\title{
A BIBLIOGRAPHY OF GENETIC RESEARCH PUBLISHED IN GERMANY, 1942-1944
}

\author{
Compiled by G. STAUDT \\ Koiser-Wilhelm-Institut für Kulturpflanzenforschung, Berlin-Dahlem
}

Received I.viii.47

The present list continues that compiled by A. Buzzati-Traverso (Heredity, I, I9-5I) and includes supplementary references to that list for the period 1939-1941.

Abbreviations : D., Drosophila.
$*$ Plant materials. $\quad+$ Microorganisms and Viruses.

Amstadt, E., 1942. Stammbaum eines Kindes mit Retinagliom. Erbarzt, Io, 2I2-2 I9.

ANDRESEN, J., 1944. Über die Vererbung von Nierenmissbildungen an Hand von Familienuntersuchungen. Z. Menschl. Vererb. u.s.w., 28, 41-8I.

Baerecke, M. L.,* 1944. Zur Genetik und Zytologie von Oenothera. Flora, $3^{8}$, $57-92$.

Barthelmess, A., 194I. Über Beziehungen zwischen Genetik, Systematik, Morphologie und Entwicklungsphysiologie und die Möglichkeit einer Zusammenarbeit. Z. ges. Naturwiss., 7, 84-92.

Barthelmess, A., ${ }^{*}$ r943. Erbgut und Gehalt an Vitamin G bei Pflanzen. Z. ges. Naturwiss., 9, $124^{-1}$ 30.

Bauch, R.,* 1942. Chemogenetische Untersuchungen an der Hefe. Ber. dtsch. Bot. Ges., 60, 42-63.

Bauch, R.,* 1943. Experimentelle Mutationsauslösung bei der Hefe durch chemische Stoffe. Zbl.f. Bakteriol., 105, 14 I.

BAuch, R., * 1944 . Die Erblichkeit der durch Radiumbestrahlung bei der Hefe ausgelösten Riesenzellbildung. Arch. Mikrobiol., I3, 352-364.

Bauer, H., 1942. Der Aufbau der Chromosomen und seine Abänderung. Jena. Z.f. Naturwiss., 75, 300-323.

BaueR, H., 1943. Die Entstehung von Chromosomen-Mutationen durch Röntgenbestrahlung. Eine Stellungnahme zu den Arbeiten von Marquardt. Z. Botanik, 38, 26-4I.

BAUer, H., 1943. Eine neue Mutation, facet-notchoid, bei $D$. melanogaster und die Deutung des notch-Effektes. Z.I.A.V., 8I, 374-39o.

Becker, H., ${ }^{*}$ 1942. Ergebnisse und Erfahrungen bei der Resistenzzüchtung gelbrostwiderstandfähiger Weizen. Z. Pflanzenzüchtg., 24, 539-568.

Beller, K., und Bieling, R., $\uparrow$ 1942. Viruskrankheiten. 2. Teil : Die Viruskrankheiten der Haus- und Laboratoriumstiere, ihre Erreger und ihre Bekämpfung. Leipzig.

Bertsch, F., * 1942. Neue vorgeschichtliche Einkornfunde aus Deutschland. Beih. Bot. Zbl., 6r, 347-35I.

Bетн, K., 1943. Entwicklung und Regeneration von Acicularia Schenkii. Z.I.A.V., $8 I, 252-270$.

Beth, K., 1943. Ein und zweikernige Transplantate zwischen Acetabularia mediterranea und Acicularia Schenkii. Z.I.A.V., 8I, 27 I-312.

BETH, K., 1943. Ein und zweikernige Transplantate verschiedener Acetabulariaceen. Naturwiss., 3I, 206-207. 
BindseIL, W., 1942. Bericht über einen Fall von erblicher Spaltbildung an Händen und Füssen. Z. Menschl. Vererb. u.s.w., 26, 357-365.

Bindseil, W., 1942. Über Papillarmuster bei einem Fall von erblicher Spaltbildung an Händen und Füssen. Z. Menschl. Vererb. u.s.w., 26, 365-376.

Bleuler, M., 1942. Theoretische und klinische Erbpsychiatrie. Naturwiss., 17, 529-540.

BöckrR, E., 1943. Zur Erblichkeit der Dysostosis multiplex. Z. Kinderheilk., 63, 688-698.

Bolsunow, I.,* 1944. Zur Untersuchung der Heterosis bei Nicotiana rustica L. Z. Pflanzenzüchtg., 26, 223-244.

BonetT, E., und IllÉNyi, A., $†$ 1943. Die Wirkung des Colchicins auf den Bakterienstoffwechsel. Z Zbl. Bakt., 105, 137.

Born, H. J., LANG, A., und Schramm, G.,* 1943. Markierung von Tabakmosaikvirus mit Radiophosphor. Arch. ges. Virusforschg., 2, 461-480.

BöRnER, C.,* 1943. Die ersten reblausimmunen Rebenkreuzungen. Z. angew. Bot., 25, 1 26-1 43 .

BörNer, C., und Gollmick, F.,* 1943. Blutlausimmune Naumburger Edelapfelzüchtungen. Z. angew. Bot., 25, 144-149.

Boutrenwex, H., 1943. Erbe und Persönlichkeit. Charakterologische Ergebnisse der Zwillingsforschung. Wien : Deuticke.

Bramstedt, F., ${ }^{*}$ 1 944 . Der Nachweis der Blutlausunanfälligkeit der Apfelsorten auf histologischer Grundlage. Z. Pflanzenkrankh. u.s.w., $48,4^{80}-488$.

Brandt, G., und Weine, F. A., 1943. Der Erbeinfluss bei Polyarthritis rheumatica (Zwillingsuntersuchungen). Z. Menschl. Vererb. u.s.w., 27, 199-223.

BrüchER, H.,* 1943. Experimentelle Untersuchungen über den Selektionswert künstlich erzeugter Mutanten von Antirrhinum majus. Z. Bot., 39, 1-48.

BRücher, H.,* 1943. Die reziprok verschiedenen Art- und Rassenbastarde von Epilobium und ihre Ursachen. III. Fb. wiss. Bot., 331-351.

Burgeff, H.,* 1943. Genetische Studien an Marchantia. Gustav Fischer, Jena.

Butenand, A., $\dagger$ 1944. Zur Feinstruktur des Tabakmosaikvirus. Abh. Preuss. Akad. Wiss. Math.-nat. Kl.

Charetschko-Savizkaja, H.,* 1944. Selbststerilität und Selbstfertilität bei Beta vulgaris L. Z. Pflanzenzüchg., 26, 103-118.

Charetschko-Savizkaja, H.,* 1944. Selektive Befruchtung. Z. Pflanzenzüchtg., $26,187-198$.

Christiansen-Wentger, F.,* 1943. Ablauf und Modifizierbarkeit des Entwicklungsrhythmus bei Weizen. Z. Pflanzenzüchtg., 25, 305-342.

Christoff, M., und Papasowa, G.,* 1943. Die genetischen Grundlagen der apomiktischen Fortpflanzung in der Gattung Potentilla. Z.I.A.V., 81, I-27.

Constantinescu, G. K., u.a., 1942. Untersuchungen über die Vererbung der Feinstrukter der Schweineborste. Z. Tierzüchtg. u.s.w., 53, 1-38.

DanneEL, R., 1943. Melaninbildende Fermente bei $D$. melanogaster. Biol. Z Zbl., $63,377-394$.

Darlington, C. D., und Wertz, E., 1942. Biologische Grundfragen in physikalischer Betrachtung. Naturwiss., 30, 250-253.

Daskaloff, C. ${ }^{*}$ 1943. Neue Ergebnisse aus Kreuzungen Sol. racemigerum $\times$ Sarya und Plowdiwer. Züchter, 15, 92-96.

v. Dellinghausen, M.,* I 944 . Zellphysiologische Untersuchungen an Epilobien mit genetisch verschiedenen Plasmen. Planta, 34, 17-34.

Dengler, A.,* 1942. Herkunfts- und Kreuzungsversuche im Versuchsgarten des Waldbauinstituts Eberswalde. Mitt. Dtsch. Dendr. Ges., 55, 157-169.

Diannelidis, T.,* 1944. Über das spontane Vorkommen von diploidem Allium carinatum. Wiener Bot. Z., 93, 66-73.

Drene, K., 1941. Das Erbe als Formgesalter der Tuberkulose. Experimente über Tuberkulose bei Kaninchen. Leipzig. 
DiEHL, K., 1942. Tierexperimentelle Erbforschung bei der Tuberkulose. Forschg. u. Fortschr., I8, 179-180.

Dillewijn, G. van, ${ }^{*}$ 1942. Fortschritte der Zuckerrohrzüchtung. Z. Pflanzenzüchtg., $24,569-59$.

Dotrerwerch, H., 1944. Die Auslösung von Mutationen durch Follikelhormon und zusätzlichem Wärmeschock bei D. melanogaster. Biol. Gen., I7, 419-446.

Dresel, E. G., und Weineck, E., 1943. Über die genetische Bedingktheit der Resistenz von Kaninchen gegenüber Lapinevirus-Infektionen. Z. Immun.Forschg., 102, 466-470.

EberHARDt, K., 1943. Ein Fall von geschlechtskontrollierter Vererbung bei Betta splendens Regan. Z.I.A.V., 8I, 72-83.

EBERHARDT, K., 1943. Geschlechtsbestimmung und -differenzierung bei Betta splendens Regan I. Z.I.A.V., 8I, 363-373.

EckHARDT, H., 1942. Über die genetische Einheit verschiedenartiger Extremitätenmissbildungen. Erbarzt, 10, 10-83.

Eмpтing, I., 1942. Systematische und erbbiologische Untersuchungen über Papillarlinien. Z. Menschl. Vererb. u.s.w., 26, 264-278.

Engström, A., 1943. Korrelation zwischen Aschengehalt und Ultraviolettabsorption bei verschiedenen Zellbestandteilen. Chromosoma, 2, 459-472.

ERnst, A.,* 1943. Kreuzungen zwischen dimorphen Primula-Arten und ihre Aufschlüsse zum Heterostylieproblem. Planta, 33, 615-636.

ERNST, S., 1942. Intrauterine Umwelt und Missbildung. Erbarzt, Io, 83-267.

Falta, W., 1943. Erbbiologie des Diabetes. Arch. inn. Med. Wien, 37, 1-26.

Fehlinger, H., 194I. Langlebigkeit und erbliche Veranlagung. Z. ges. Naturwiss., $7,350-352$.

Fischer, W., 1942. Beitrag zur Vererbung der Blutgruppen, Untergruppen und Blutkörperchenmerkmale. Erbarzt, Io, 242.

FrrTke, H., 1943. Über eine ungewöhnliche Form "Multipler Erbabartung." Z. Kinderheilk., 63, 5 10-523.

Freisleben, R.,* 1943, Ein neuer Fund von Hordeum agriocrithon Åberg. Zü̈chter, $15,25-29$.

Freisleben, R., und Lein, A.,* 1943. Vorarbeiten zur züchterischen Auswertung röntgeninduzierter Mutationen. Z. Pflanzenzüchtg., 25, 235-254.

Freisleben, R., und Lein, A., ${ }^{*}$ 1943. Mutationen des Chlorophyllapparates als Testmutationen für die mutationsauslösende Wirkung der Bestrahlung bei Gerste. Z. Pflanzenzüchtung, 25, 255-283.

Freisleben, R., und Lein, A., * 1944 . Möglichkeiten und praktische Durchführung der Mutationszüchtung. Kühn-Arch., 60, $211-225$.

Frex-Wyssling, A., 1943. Doppelbrechung und Dichroismus als Mass der Nucleinsäure-Orientierung in Chromosomen. Chromosoma, 2, 473-481.

v. Frimmel, F., ${ }^{*}$ 1943. Welcher Artbildungsvorgang hat zur Domestikationsform Lycopersicum esculentum Mill. geführt? Z. Pflanzenzüchtg., 25, 437-442.

Geipel, G., 1943. Zur Methode der Ermittlung der Genformeln der Fingerleisten im Vaterschaftsnachweis. Leipzig.

Geitler, L., 1943. Die Bedeutung der Endomitose für die Krebscytologie und Jacobys "heterotyp-genmutative" Geschwulsttheorie. Roux' Arch., I42, 301-310.

Gertler, L., ${ }^{*}$ r 943 . Über eine postmeiotische Teilsungsanomalie und den Spiralbau der Chromosomen von Paris quadrifolia. Chromosoma, 2, 519-530.

Geitler, L.,* 1943. Fruchtformen der Hasel in Wildpopulationen. Wiener Bot. Z., 92, 87-93.

GeItrer, L., 1943. Die Mechanik der Mitose. Naturwiss., 31, 50I-504.

Gertrer, L.,* 1943. Natürliches diploides Allium carinatum. Ber. dtsch. Bot. Ges., $61,210-211$.

Geitler, L., 1944. Zur Kenntnis des Kern- und Chromosomenbaus der Heuschrecken und Wanzen. Chromosoma, 2, 531-543. 
Gertler, L.,* I944. Der Bau der Riesenkerne des Elaiosoms von Corydalis cava. Chromosoma, 2, 544-548.

Gloor, H., und HADORN, E., I942. Vergleich der Schädigung im Ovar von D. melanogaster, bewirkt durch einen Letalfaktor und ein Sterilitätsgen. KühnArch., 6o, 438-440.

Gobs-Sonnenschein, C., ${ }^{*}$ I943. Die experimentelle Erzeugung polyploider Sojabohnen mit Alkaloidgemischen in Verbindung mit Kreuzungen polyploider Rassen. Züchter, 15, 62-68.

GöHRE, E., 1942. Untersuchungen über den plasmatischen Feinbau der Gregarinen mit besonderer Berücksichtigung der Sexualitätsverhältnisse. Arch. Protistenk., $96,295-325$.

Gollmick, F.,* 1942. Über die Lebensdauer des Rebenpollens. Angew. Bot., 24, H. I u. 2.

Göthlin, G. F., I942. Die Erblichkeit angeborener totaler Farbenblindheit mit Lichtscheu. Erbarzt, to, 2 I8.

Göтz, B., 1942. Zum Geschlechtsverhältnis der Traubenwickler. Z. angew. Entomologie, 29, 313-329.

Gotrschaldt, K., 1942. Die Methodik der Persönlichkeitsforschung in der Erbpsychologie. Leipzig.

Grebe, H., 1942. Über die Differentialdiagnose und Erbverhältnisse beim primordialen Zwergwuchs. Erbarzt, 10, 195-2 I0.

Grebe, H., I942. Dysplasie der rechten Körperhälfte bei einem Paarling von eineiigen Zwillingsschwestern. Erbarzt, Io, 99-126.

Grebe, H., I942. Die Fistula sacrococcygea, ein Erbmerkmal. Erbarzt, ro, I 23- 95 .

Grebe, H., 1942. Gibt es eine erbliche Struma? Erbarzt, ro, 278-282.

Grebe, H., 1944. Beitrag zur Ätiologie des Situs inversus. Z. Menschl. Vererb. u.s.w., $28,262-272$.

Grebe, H., I944. Die Akrocephalosyndaktylie. Eine klinisch-ätiologische Studie. Z. Menschl. Vererb., 28, 209-262.

GreIs, H., I942. Mutations- und Isolationsversuche zur Beeinflussung des Geschlechts von Sordaria fimicola (Rob.) III. Z. Bot., 37, 1-109.

Griesinger, R.,* I942. Die Bedeutung der Ergebnisse der Polyploidieforschung für die Pflanzenzüchtung. Ber. dtsch. bot. Ges., 6o, 36-4I.

Gruhn, R., 1942. Theoretische Grundlegung der Eltern-Nachkommenkorrelation. I. und II. Z. Tierzüchtg., 53, 89-144, 227-267.

Grunn, R., 1944. Die Elternnachkommenkorrelation bei Koppelung und multipler Allelie. Z. Tierzüchtg., 56, 261-298.

GüNTHER, R., 1943. Über erbliche Gehörgangsatresie. Z. Menschl. Vererb., 27, 253-269.

HAAK, D., 1942. Untersuchungen an eineiigen und zweieiigen Rinderzwillingen hinsichtlich der Ähnlichkeit morphologischer und physiologischer Merkmale. Züchtgskunde, $17,326-330$.

HaARDT, K., 1943. Uber die Ausprägungsweise und Ausprägungsbedingungen der Mutationen Kfe (kurzflüglig) bei Ephestia kühniella Z. Biol. Zbl., 63, 478-487.

Hadorn, E., und Zeller, H., 1942. Mutteralter und Fertilität bei Drosophila. Kühn-Arch., 6o, 440-444.

Hadorn, E., und Zeller, H., 1943. Fertilitätsstudien an D. melanogaster. I. Untersuchungen zum altersbedingten Fertilitätsabfall. Roux' Arch., r42, 276-300.

Hämmerling, J., ${ }^{*}$ 1943. Ein und zweikernige Transplantate zwischen Acetabularia mediterranea und A. crenulata. Z.I.A.V., 8I, I I4- 180 .

Hämmerling, J.,* 1943. Entwicklung und Regeneration von Acetabularia crenulata. Z.I.A.V., $81,84^{-1} 13$.

Harte, C., ${ }^{*}$ I943. Meiosis und Crossing-over. Weitere Beiträge zur Cytogenetik von Oenothera. Z. Bot., 38, 65-137. 
Hartmair, V.,* r943. Colchicinininduzierte Polyploidie bei Gurken. Züchter, $15,13-16$.

Hartmann, M., 1943. Die Sexualität. G. Fischer, Fena.

Heberer, G., 1944. Die Chromosomenverhältnisse des Menschen im Lichte der Cytogenetik. Fena. Z. Med. u. Naturwiss., 77, $15^{1-1} 7^{78}$.

Herbst, C., 1943. Die Bedeutung der Salzversuche für die Frage nach der Wirkung der Gene. Zusammenschau einer Hydrations-bzw. Microlaboratorienbautheorie der Genwirkungen. Roux' Arch., I42, 319-378.

Hertwig, G., i 943 . Das Volumen der Zellkerne und die Zahl und Grösse der Nucleolen haploider, diploider und triploider Froschlarven verschiedenen Alters. Z. mikrosk.-anat. Forschg., 53, 445-466.

Hertwig, P., 1944. Die Genese der Hirn- und Gehörorganmissbildung bei röntgeninduzierten Kreisler-Mäusen. Z. Menschl. Vererb., 28, 327-355.

HiRschleR, J., 1942. Über perinukleäre Membranen in den männlichen Geschlechtszellen einiger Lepidopteren-Arten. Biol. Zbl., 62 .

Hoffmann, W.,* 1944. Die Vererbung der Winter-Sommer-Form und Winterfestigkeit der Gerste. Z. Pfanzenzüchtg., 26, 56-9r.

HofstÄtter, P. R., r943. Die Faktorenanalyse somatischer Merkmale. Z. Menschl. Vererb., 27, 579-603.

HoLst, H., 1944. Ưber die Häufigkeit und Vererbung der Schuppenflechte. Z. Menschl. Vererb., 28, $4^{\text {18-437. }}$

Husfeld, B., ${ }^{*}$ 1943. Die züchterischen Möglichkeiten in der Menge und Güte des Ertrages bei interspezifischen Vitis-Kreuzungen, Wein u. Rebe, 25, 4-28.

IliefF, P.,* 1942. Genetische Untersuchungen an der Erdnuss (Arachis hypogaea L.) Bohnenfarbe und Fruchtgrössenvererbung. Züchter, 14, 141-145.

JACOBY, W.,* 1943. Zur Aussprache mit Geitler über die heterotyp-genmutative Entstehung der bösartigen Geschwülste. Roux'Arch., I42, 31 I-318.

KAPPERT, H.,* 1944. Untersuchungen über Plasmonwirkungen bei Aquilegia. (Gynodioecie-Heterosis-Gestalt des Sporns.) Flora, Fena, 37, 95-105.

KeIL, R., I943. Der Kaliumgehalt der D. melanogaster (Wildform) und die spontane Mutabilität. Biochem. Z., 313, 317-336.

KLATT, B., r 943 . Kreuzungen an extremen Rassentypen des Hundes. III. Der Bulldoggschädel und die Frage der Chondrodystrophie. Z. Menschl. Vererb., 27, 283-346.

KLATT, B., 1944. Kreuzungen an extremen Rassentypen des Hundes. IV. Schädel und Physiognomie der Kreuzungstiere. Z. Menschl. Vererb., 28, I $13-159$.

KLATt, B., 1944. 3o Jahre Inzucht beim Schwammspinner. Zool. Anz., 144, 169-184.

Klawitter, G., und v. Sengbusch, R.,* 1943. Die Züchtung von vollkommen alkaloidfreien Süsslupinen, die sich zur Herstellung von menschlichen Nahrungsmitteln eignen. Züchter, 15 , 10-12.

KnapP, E.,* 1943. Bemerkungen zu Geschlechtsbestimmungsfragen. Flora, Fena, $37,139-151$.

KNAPP, E.,* 1943. Ist das heterochromatische X-Chromosom von Sphaerocarpus in seiner ganzen Länge lebensnotwendig? Naturwiss., 3I, 570.

KöHLER, E., 1943. Die entwicklungsphysiologische Korrelation zwischen Farbe, Form, und Struktur der Schmetterlingsschuppen in der Genese des Zeichnungsmusters. Naturwiss., 3I, $128-135$.

Konsuloff, S., 1943. Ein Erbmerkmal auf der Fersenhaut des Menschen. Z. Menschl. Vererb., 27, 76r-765.

Kostoff, D.,* r 943 . Haploide Triticum vulgare und die Variabilität ihrer diploiden Nachkommenschaften. Züchter, ${ }_{5}$, 121-125.

KötschaU, K., 1942. Anlage oder Umwelt? Erbarzt, 1o, 253-258.

KŘ́ľ̌NECKÝ, J., r944. Versuch einer Ableitung allgemeiner Formeln für die Anzahl möglicher Kombinationen und verschiedener Genotypenarten beim Polyhybridismus bei beliebigen Genotypen der Eltern. Z. Tierzüchtg., 57, 1-10. 
KüHN, A., 1942. Über eine Genwirkkette der Pigmentbildung bei Insekten. Nachr. Ges. Wiss., Göttingen Math.-phys. Kl.

KüHN, A., I942. Über eine zu d. $f_{1}$ isophäne autosomale Mutation bei Ephestia kühniella Z. Biol. Zbl., 62, 550-554.

KüHN, A., 1943. Prädetermination von a-Merkmalen durch Kynurenin. Abh. preuss. Akad. Wiss. Math.-nat. Kl., $9, \mathrm{I}-6$.

KüHN, A., 1944. Eine neue Mutation der Schuppenpigmentierung (ca) bei $P_{t y \text { - }}$ chopoda seriata Schrk. Biol. Zbl., 64, 154-157.

KüHN, A., 1944. Über eine die Schuppenformbildung und Schuppenpigmentierung beeinflussende Mutation (vd) von Ephestia kühniella Z. Biol. Zbl., 64, 81-97.

Kühn, A., und Schwartz, V., I942. Über eine weissäugige Mutante von Ephestia kühniella. Biol. Zbl., 62, 226-230.

Kühn, A., und v. Engelhardt, M., 1943. Über zwei melanistische Mutationen (At und ni) von Ptychopoda seriata Schrk. Biol. Zbl., 63, 251-267.

Kühn, A., und v. Engelhardt, M., 1943. Über zwei Entschuppung bewirkende Mutationen (Vi und cal) bei Ptychopoda seriata. Biol. Zbl., 63, 470-478.

KüHN, A., und v. Engelhardt, M., I 944 . Mutationen und Hitzemodifikationen des Zeichnungsmusters von Ptychopoda seriata Schrk. Biol. Zbl., 64, 24-73.

Kunn, R., Löw, I., und Moewus, F., 1942. Über die Wirkungsweise eines geschlechtsbestimmenden Stoffes (Borsäure). Naturwiss., 3o, 407-408.

KunHK, R.,* 1943. Vergleichende Untersuchungen an di- und tetraploidem Lein (Linum usitatissimum L.). Z. Pflanzenzüchtg., 25, 93-I I I.

LABERKE, J. A., r944. Über Rassenmerkmale, Bauschwächen und Abnutzungskrankheiten in einem Insuchtsippenkreis. Z. Menschl. Vererb., 28, I59-167.

LANG, A., und Melchers, G., * 1943. Die photoperiodische Reaktion von Hyoscyamus niger. Planta, 33, 653-702.

LehmanN, E.,* 1942. Zur Genetik in der Gattung Epilobium. IV. 7b. wiss. Bot., 9o, 49-99.

LehmanN, E.,* 1944. Zur Genetik der Entwicklung in der Gattung Epilobium. V. Das Verhalten der Tübinger Biotypen von Epilobium hirsutum bei Einkreuzungen mit E. adenocaulon. Jb. wiss. Bot., 9I, 439-502.

Lehmann, W., und Heilmann, K., I943. Beitrag zur Erbpathologie der splenomegalen Cirrhosen. Z. Menschl. Vererb., 27, 20-39.

LeIN, A., ${ }^{*}$ I 943 . Die genetische Grundlage der Kreuzbarkeit zwischen Weizen und Roggen. Z.I.A.V., 8r, 28-6r.

LeIN, A.,* 1943. Die Wirksamkeit von Kreuzbarkeitsgenen des Weizens in Kreuzungen von Roggen o mit Weizen $\delta$. Züchter, 15, I-3.

LeIN, A.,* r944. Ủber Rückkreuzungsversuche eines amphidiploiden Weizen $\times$ Roggenbastards mit Rogen. Kiuhn-Arch., 6o, 226-237.

Letrré, H., 1943. Mitosegifte aus der Steringruppe. Hoppe-Seylers Z., 278, 206-207.

LetreE, H., 1943. Hemmung der Zellteilung durch oestrogene Faktoren. HoppeSeylers Z., 278, $201-205$.

LertrE, H., 1943. Zur Teilungsgeschwindigkeit der Zellen des Mäuse-AscitesTumors. Naturwiss., 31,467 .

LiEbenam, L., 1942. Zwei neue Merkmale zur Zwillingsdiagnose. Umschau, 42, $211-212$.

Liebenam, L., 1942. Über gleichzeitiges Vorkommen multipler Missbildungen. Z. Menschl. Vererb., 26, 242-257.

Ludwig, W., 1942. Über die Rolle des Mutationsdruckes bei der Evolution. Biol. Zbl., 62, 374-379.

LudWIG, W., I942. Zur evolutionischen Erklärung der Höhlentiermerkmale durch Allelelimination. Biol. Zbl., 62, 447-454.

v. MARCK, E., 1943. Über die Kern-Plasma-Relation differenzierter Gewebezellen : Vorkommen und Grösse. Z. Zellf. Mic. Anat., 32, 557-586.

MarquardT, H.,* 1943. Zur Analyse röntgeninduzierter Chromosomenveränderungen und Chromosomenmutationen. Eine Erwiderung. Z. Bot., 38, 42-63. 
Marquardt, H., * 1943. Über eine röntgeninduzierte Mutation mit abweichendem Erbverhalten bei Oenothera Hookeri. Flora, Fena, 37, 152-165.

Mell, R., 1942. Experimentelle Untersuchungen über die geographische Verbreitung von Plasmon-Unterschieden und der auf diese Unterschiede empfindlichen Gene, sowie deren theoretische Bedeutung für das Kern-PlasmaProblem. Biol. Zbl., 62, 170-186.

Mrchaelis, P., 1943. Untersuchungen an reziprok verschiedenen Artbastarden bei Epilobium. I u. 2. Flora, Fena, 37, 1-56.

Moewus, F., ${ }^{*}$ 1943. Statistische Auswertung einiger physiologischer Versuche an Protosiphon und Chlamydomonas. Biol. Zbl., 63, 169-203.

Möglich, F., Rompe, R., und Timoféeff-Ressovsky, N. W., 1944. Energieausbreitungsmechanismen in Physik und Biologie. Forschg. u. Fortschr., 20, 6-10.

DE MoL, W. E.,* 1942. Fortgesetzte Untersuchungen betreffs somatischer Tulpenmutationen, u.s.w. Gartenbauziss., 16 , 70-9o.

DE MoL, W. E.,* 1943. Die früher blühenden somatischen Tulpenmutanten und die Teilungshypothese. Gartenbauwiss., 17 , I06-1 32 .

DE MoL, W. E., * 1943 . Die erste gezüchtete triploide Scilla sibirica Andrews. Gartenbauwiss., $17,227-240$.

MÜNDleR und Schwanitz,* 1942. Über einen Ertrags- und Düngungsversuch mit diploidem und autotetraploidem Münchner Bierrettich. Züchter, 14, 137-140.

Nachtsheim, H., 1943. Die Pelger-Anomalie und ihre Vererbung bei Mensch und Tier. II. Die homozygoten Pelger und ihr Schicksal. Erbarzt, II.

Nachtsheim, H., 1943. Das Problem der Verwilderung von Haustieren. Forschg. $u$. Fortschr., 19.

Neugebauer, Th., $\dagger$ 1942. Über die autokatalytische Entstehung der Viren und verwandte Probleme. Naturwiss., 30, 168.

NovÁ, J., 1943. Genetische Polynome als Grundlage zur Ableitung der Genotypen und Phänotypen, u.s.w. Z.I.A.V., $8 I, 343-362$.

OeHLKERs, F.,* I 942 . Bastardierungsversuche in der Gattung Streptocarpus Lindb. Z. Bot., 37, 158-182.

OenlKers, F., * 1943. Die Auslösung von Chromosomenmutationen in der Meiosis durch Einwirkung von Chemikalien. Z.I.A.V., 81, 313-341.

OenlKers, F.,* 1944. Cytologische und cytogenetische Untersuchungen an Streptocarpus. Z. Bot., 39, I 13-16o.

OehlKers, F., und Harte, C.,* ${ }^{*}$ r 943 . Über die Aufhebung des Gonen- und Zygotenausfalls bei Oenothera. Flora, Jena, 37, 106-1 24.

Oxenius, K., 1942. Zur Frage der erblichen Bedungtheit der Mehrlingsgeburten. Münch. Med. Wchschr., 44.

Pascher, A.,* 1943. Beiträge zur Morphologie der ungeschlechtlichen und geschlechtlichen Vermehrung der Gattung Chlamydomonas. Beih. z. bot. Zbl., A $62,197-220$.

Pätau, K., 1943. Zur statistischen Beurteilung von Messungsreihen. (Eine neue T-Tafel.) Biol. Zbl., 63, 152-169.

Pätau, K., I944. Das Wrightsche Modell der Evolution. (Genetische Theorie der Evolution der Organismen 1.) Naturwiss., 32, 196-202.

Pätau, K., und Timoférff-Ressovsky, N. W., 1943. Statistische Prüfung des Unterschiedes der Temperaturkoeffizienten hoher und normaler Mutationsraten, u.s.w. Z.I.A.V., $81,62-72$.

Pause, F., und Gierlich, J., 1942. Konkordante Bauchspalte bei eineiigen Zwillingen. Z. Menschl. Vererb., 28, 399-4I8.

Persch, R., 1942. Über erbliche doppelseitige Athetose besonders bei Geschwistern. Erbarzt, $10,49-66$.

Pfaff, W., 1942. Das Erbe als Formgestalter der Tuberkulose. Dtsch. med. Wchschr., 68, $201-202$.

Prppow, G., 1942. Über das Zusammentreffen von Wirbelgelenkaplasien und Brachydaktylie in einer Sippe. Erbarzt, 10, 276-277. 
Prigge, R., und v. Schelling, H. 1942. Zur Analyse der Antigenwirkung. Naturwiss., 30, 66r.

Pterová. J., I942. Über den Vergleich der $\alpha$-Strahlenempfindlichkeit von Kern und Plasma. Ber. disch. Bot. Ges., 6o, I48-1 5I.

Renner, O.,* ז943. Zur Kenntnis des Pollenkomplexes flectens der Oenothera atrovirens Sh. et Bartl. Z.I.A.V., 8r, 391-483.

RenNer, O.,* 1 943 . Über das crossing-over bei Oenothera. Flora, Jena, 36, i 1 7-2 14.

Renner, O.,* 1943. Die Variabilität der Oenothera biennis L. Mitt. Thür. Bot. Ver. N.F., 50, $14^{-1} 9$.

Renner, O.,* I 943 . Kurze Mitteilungen über Oenothera. V. Zur Kenntnis von O. silesiaca n. sp., parviftora L., ammophila Focke, rubricaulis Kleb. Flora, Fena, $36,325-334$.

RenNer, O.,* ${ }^{*}$ I943. Über die Entstehung homozygotischer Formen aus komplexheterozygotischen Oenotheren. II. Die Translokationshomozygoten. Z. Bot., 39, 49-1 06 .

RenNer, O.,* 1944. Kurze Mitteilungen über Oenothera. VI. Über die I5-chromosomigen Mutanten dependens, incana, scintillans, glossa, tripus. Flora, Jena, 37, $216-229$.

RENSCH, B., 1943. Die paläontologischen Evolutionsregeln in zoologischer Betrachtung. Biol. Gen., 17, I-55.

Rietschel, P., 1942. Über die Zunahme der Gleicherbigkeit durch fortgesetzte Inzucht und Auslese bei der Honigbiene. Z angew. Entomol., 28, 571-583.

Roemer, Th.,* I 942-43. Handbuch der Pflanzenzüchtung. Bd. 3. Gräsersüchtung. Brassica Futterpflanzen. Register Verl. Parey, Berlin.

Rosendahl, G.,* 1944 . Cytologische Untersuchungen an tetraploiden Gersten. Kühn-Arch., 6o, 238-252.

v. Rosenstiel, K., und Mrttelstenscheid, L.,* i943. Über die Erzeugung amphidiploider Roggen-Weizenbastarde (Secalotrica). Züichter, 15, 1 73-183.

Ross, H., ${ }^{*}$ 1943. Über die Verschiedenheiten des dissimilatorischen Stoffwechsels in reziproken Epilobium-Bastarden, u.s.w. Planta, 33, I6 $1-184$.

Rössger, W., 1942. Beitrag zur Technik der Weizenkreuzung. Forsch.-dienst, 14, 330-334.

Rothmaler, W.,* 1943. Zur Gliederung der Antirrhineae. Feddes Repertorium, $52, \times 6-39$.

Routrl, R., 1942. Ein Beitrag zur Frage der Vererbung der Schädelform. $Z$. Morph. u. Anthrop., 40, 80-9I.

DE Rudder, B., r943. UUber" Phosphatiddiathese " und ihr Verhältnis zu Dysostosis multiplex und Dysostosis Morquio. Eine klinischerbbiologische Analyse. Z. Kinderheilk., $63,407-422$.

Rudorf, W.,* 1943. Die Bedeutung der Polyploidie für die Evolution und die Pflanzenzüchtung. Angew. Bot., 25, 92-I I 4.

Sartorius, O.,* 1942. Vererbungsstudien an der Weinrebe mit besonderer Berücksichtigung des Blattes. Gartenbauwiss., $16,12-24$.

v. Schelring, H., 1943. Zur Deutung auslesefreier Zwillingserhebungen. Z. Menschl. Vererb., $27,778-782$.

Scherz, W.,* 1943. Ein Weg zur Züchtung gegen Plasmopara viticola resistenter Rebenformen durch Auffinden somatischer Mutanten innerhalb der Species Vitis vinifera. Züchter, 15, 205-2 10.

Schiemann, E.,* 1939. Gedanken zur Genzentrentheorie Vavilovs. Naturw., $27,377-383,394-40$ I.

Schizmann, E.,* 1940. Kritisches zur Datierung alter Getreidefunde. Prähist. Z., $30,3-34,371-378$.

SchirmanN, E.,* 1940. Die Biologisch-genetische Abteilung des Botanischen Gartens in Dahlem. Notizbl. d. Bot. Gart. u. Mus., ${ }_{5}$, I-18.

Schiemann, E.,* 1940. Die Getreidefunde der neolithischen Siedlung Trebus, Kr. Lebus/Mark. Ber. disch. bot. Ges., $5^{8}, 44^{6-459}$. 
Schiemann, E.,* 1943. Artkreuzungen bei Fragaria III. (1. Teil). Die vescaBastarde. Flora, Jena, 37, 166-192.

Schiemann, E.,* 1943. Entstehung der Kulturpflanzen. Ergebn. Biol., 19, $412-55^{2}$.

Schlösser, L. A., * 1944 . Über das Fertilwerden autoploider Leinsippen. Züchter, I6, 3-8.

Scнmidt, M.,* 1942. Ein Fall gehäufter Chimärenbildung bein $\Lambda$ pfel. Züchter. I4, $112-117$.

Schmidt, C., und Sauermann, H., 1943. Häufigkeit und Krankheitserwartung des Diabetes mellitus, u.s.w. Z. Menschl. Vererb., 27, 443-508.

Schramm, G., und Rebensburg, L., $\dagger$ 1942. Zur vergleichenden Charakterisierung einiger Mutanten des Tabakmosaikvirus. Naturwiss., 3o, 48-5I.

Scrröck, O.,* 1943. Genetische Beobachtungen an Luzerne (Medicago media). Z. Pflanzenzüchtg., 25, 81-91.

Schröck, O.,* 1943 . Beobachtungen an einem Bastard zwischen Luzerne (Medicago media) und Gelbklee (Medicago lupulina) und seiner Nachkommenschaft. Züchter, ${ }_{15}, 4-9$.

Schröck, O.,* 1944. Untersuchungen an diploiden und tetraploiden Klonen von Luzerne, Gelbklee und Steinklee. Z. Pflanzenzüchtg., 26, 2 1 4-222.

Schüтze, R., 1943. Die Beeinflussung der Mutationsrate bei $D$. melanogaster durch kombinierte Behandlung mit Arsen und Röntgenstrahlen. Z.I.A.V., 8I, 484-499.

Schwarz, O., r944. Eine neue Mutation des Zeichnungsmusters (us) bei Ephestia kühniella. Biol. Zbl., 64, 305-315.

Schwemmele, J., * 1943. Plastiden und Genmanifestation. Flora, Fena, 37, 61-72.

Schwier, H., 1943. Vitalitätsuntersuchungen an normalen und albinotischen Makropoden. Zool. Anz., I43, 33-44.

Schwier, H., 1943. Über die Vererbung des Streifenmusters in der Gattung Macropodus. Zool. Anz., I4I, I 79-24I.

SeEliger, R., 1943. Genetische Untersuchungen an dem Flügelmuster des Bohnenkäfers Zabrotes subfasciatus Boh. (Coleopt. Bruchidæ). Z.I.A.V., 8I, 196-25I.

Sell-Beleites, I., und CAtsch, A., 1943. Mutationsauslösung durch ultraviolettes Licht bei Drosophila. Z.I.A.V., 80, $55^{1-557 .}$

v. Sengbusch, R.,* 1942. Methode zur züchterischen Bearbeitung von Fremdbefruchtern und anderes. Forschgsdienst, Sonderh., I6, 356-361.

v. Sengbusch, R., Freisleben, R., und Müller, E. W., ${ }^{*}$ i 943 . Röntgenologische Untersuchungsmethode von Pflanzen und Pflanzenteilen für züchterische Zwecke. Züchter, 15 .

v. Sengbusch, R., und Klawitter, G., * 1943. Zielsetzung und Probleme in der Speisemöhrenzüchtung. Züchter, ${ }_{15}, 16-22$.

v. Sengbusch, R., und Neuer, H., ${ }^{*}$ 1943. Die Geschlechtsvererbung bei Hanf und die Züchtung eines monöcischen Hanfes. Züchter, 15, 49-62.

Sirks, M. J., ${ }^{*}$ 1943. Die Möglichkeit der Kreuzung zwischen Solanum tuberosum of und Solanum chacoënse đ.' Zü̈hter, ${ }_{15}$.

v. Skramlik, E., 1943. Vererbungsforschungen auf dem Gebiet des Geschmacksinnes. Fena. Z. Med. u. Naturwiss., 76, 50-79.

Steffan, P., 1942. Die Verteilung der Blutgruppengene in Mitteleuropa. Z. Rassenphysiol., I2, 73-95.

Steingacher, G. J., i 943 . Über die Entstehung und das Alter von Vogelrassen. Zool. Anz., I4I, $14^{1-1} 79$.

StEINiger, F., und VerT, G., I 942. Über Heteropenetranz und Zwillingsdiskordan $~$ bei der erblichen Hasenscharte. Z. Menschl. Vererb., 26, 75-93.

Stelzner, D., $\dagger$ 1942. Zur Frage der Virusübertragung durch Samen, insbesondere des X-, Y- und Blattroll-Virus der Kartoffel. Züchter, I4, 225-234.

Stelzner, G., ${ }^{*}$ 1943. Wege zur züchterischen Nutzung des Solanum chacoense Bitt. in Hinblick auf die Züchtung käfferesistenter Kartoffelsorten. Züchter, 15, 33-38. 
Stelzner, G.,* 1943 . Übcr die Fertilitätsverhältnisse bei Bastardierungen zwischen der frostfesten Wildkartoffel Solanum acaule Bitt. und der Kulturkartoffel $S$. tuberosum L. Züchter, 15, I43-145.

Stomps, Тн. J.,* 1942 . Über die künstliche Herstellung von Oenothera Lamarckiana gigas de Vries. Ber. disch. bot. Ges., 6o, 125-131.

Strugger, S., 1942. Die Fluoreszensmikroskopie im Dienste der biologischen Forschung. Forschg. u. Fortschr., 18, 332-334.

Strunz, K., i944. Die mathematische Behandlung qualitativer Merkmalskopplungen mit Hilfe der Wertigkeitsmethode. Z. Menschl. Vererb., 28, 313-327.

Stubbe, H., 1942. Mutation und Art-Entstehung. Umschau, 46, I I6-1 I8.

Suchalla, H., 1943. Variabilität und Erblichkeit von Schädelmerkmalen bei Zwerg- und Riesenrassen; dargestellt an Hermelin- und Widderkaninchen. Z. Morph. Anthrop., 40, 274-333.

SunG-YUN, MA., I943. Experimentelle Untersuchungen über Hitzemodifikationen des Flügels bei D. melanogaster. Roux' Arch., 142, 508-618.

Tischler, G., ${ }^{*}$ 1942. Allgemeine Pflanzenkaryologic. 2. Hälfte: Kernteilung und Kernverschmelzung. Bornträger, Berlin.

Trendelenburg, W., I $94^{2}$. Über Vererbung bei einem fall von anormalem Farbensinn des einen und normalem Farbensinn des anderen Auges beim Mann. Merkbl. Augenheilk., 107, 280.

'Troll, H. J.,* 1943. Anthozyanmutanten bei Lupinus angustifolius L. Züchter, $15,73-78$.

Tschermak, E., ${ }^{*}$ ig43. Vergleichende und experimentelle cytologische Untersuchungen an der Gatttung Oedogonium. Chromosoma, 2, 493-518.

Tschermak, E.,* 1943. Über die Grössenverhältnisse von univalenten und bivalenten Rassen und das Auftreten natürlicher bivalentcr Rassen bei Oedogonium. Biol. Zbl., 63, 457-467.

v. UBISCH, G., I 942 . Die Bedeutung der neueren experimentellen Embryologie und Genetik für das Evolutions-Epigeneseproblem. F. Ambrosius Barth, Leipzig.

UlLRICH, O., 1943. Über polyphäne und pleiotrope Genwirkungen in der Erbpathologie des Menschen. Z. Menschl. Vererb., 27, 651-682.

v. Verschuer, O., I942. Rezessiver oder dominanter Erbgang des Diabetes. Erbarzt, 10, 67-72.

Wanner, H.,* I 943 . Zur Karyologie der Gattung Primula L. Sect. Auricula Duby. Planta, 33, 637-652.

Weber, W., 1942. Beitrag zur Methode, Statistik und Erblichkeit der Geschmacksempfindung für Phenylthiocarbamid. Erbarzt, 10, I 54-167.

Weisswange, W. M. H., 1942. Erbbiologische Beobachtungen bei der Volksröntgenuntersuchung. Erbarzt, 1o, 243-248.

WEITZ, W., 1942. Zur Methodik der Erbforsehung bei inneren Krankheiten unter besonderer Berücksichtigung der Zwillingsforschung. Z. Menschl. Vererb., 26, 289-309.

WeRTH, E.,* r 942 . Neues und kritisches zur Kenntnis alter Kulturpflanzen. Ber. dtsch. bot. Ges., 6o, 232-258.

Wolfslast, W., 1943. Eine Sippe mit recessiver geschlechtsgebundener spastischer Diplegie. Z. Menschl. Vererb., 27, I89-199.

Zimmer, K., r944. Statistische Ultramikrometrie mit Röntgen-, Alpha- und Neutronenstrahlen. Forschg. u. Fortschr., 20, 106-1 o8. 